\title{
Tensile Properties of die-cast magnesium alloy AZ91D at high strain rates in the range between $300 \mathrm{~s}^{-1}$ and $1500 \mathrm{~s}^{-1}$
}

\author{
I. R. Ahmad ${ }^{a}$, SHU D. Wei ${ }^{b}$ \\ ${ }^{a, b}$ School of Mechanical and Aerospace Engineering, Nanyang Technological University, \\ Nanyang Ave., 639798 Singapore \\ a iram0002@ntu.edu.sg, ${ }^{\mathrm{b}}$ mdshu@ntu.edu.sg
}

\begin{abstract}
Keywords: Hopkinson bar; magnesium alloys; AZ91D alloy; high strain rate; tensile properties;
\end{abstract} strain rate sensitivity

\begin{abstract}
Magnesium alloys have been increasingly used in the automobile, aerospace and communication industries due to their low density, high strength to weight ratio, good impact resistance and castability. Magnesium alloys, previously not used in load bearing components and structural parts are strongly being considered for use in such applications. Impact events in vehicles and airplanes as well as developments in weaponry and high speed metal working are all characterized by high rates of loading. Understanding of the dynamic behaviour of materials is critical for proper design and use in different applications. In the current study, a cast magnesium alloy AZ91D has been investigated at quasi-static and higher strain rates in the range between $300 \mathrm{~s}$ ${ }^{1}$ and $1500 \mathrm{~s}^{-1}$. The INSTRON machine was used to perform the quasi-static tests. High strain rate tests have been performed using the Split Hopkinson Tensile Bar (SHTB), a very useful and widely used tool to study the dynamic behaviour of variety of engineering materials. The results of a tensile testing indicate that the tensile properties including yield strength (YS), ultimate tensile strength (UTS) and the elongation at fracture $\left(E_{f}\right)$ are affected by the strain rate variation. Higher stresses are associated with higher strain rates. The alloy AZ91D displays approximately $45 \%$ higher tensile stresses at an average strain rate of approximately 1215/s than at quasi-static strain rate. The dependence of the yield stress and tensile strength on the strain rate in the range of high strain rate above $1000 \mathrm{~s}^{-1}$ is larger than that at lower strain rates. The alloy AZ91D is observed to be more strain rate sensitive for strain rate higher than $1000 \mathrm{~s}^{-1}$. A decrease in the strain rate sensitivity is also observed with the increasing strain in the specimen. It is observed that the hardening behaviour of the alloy is affected with increasing the strain rate. At high strain rates, the fracture of magnesium alloy AZ91D tends to transit from ductile to brittle.
\end{abstract}

\section{INTRODUCTION}

Light weight magnesium alloys due to their excellent strength to weight ratio and good impact strength are being investigated for use in the automotive, aerospace and electronics industries. AZ91D is being used extensively for manufacturing of gearboxes, steering column holders and brackets, cam covers, transmission housings, drive brackets, 4-wheel drive transfer cases, clutch and brake pedals and many other components. In recent years, AZ91D due to its suitable properties is also being used for information and communication technology (ICT) appliances such as mobile phones, cameras, laptops and compact and mini disc cases as well [1].

Magnesium alloys, which are rapidly becoming popular for fabricating structural and load bearing parts in the automobile, aerospace and electronic industries due to light weightiness, high specific strength and good impact resistance, have not been tested adequately at impact rates of loading. Lack of sufficient and conclusive data about their dynamic behaviour has steered the research to investigate these alloys at high strain rates. Aune et al [2] investigated the effects of strain rate on the dynamic properties of the die cast AZ91D, AM60B and AM50A. They found that the stress increases and percentage elongation is not affected by strain rates from $15 \mathrm{~s}^{-1}$ to $130 \mathrm{~s}^{-1}$. No significant strain rate sensitivity variations were found for strain rate change from $15 \mathrm{~s}^{-1}$ to $130 \mathrm{~s}^{-1}$. Ishikawa, Watanabe and Mukai [3] examined the compressive properties in the solution treated AZ91 at a strain rate of $10^{3} \mathrm{~s}^{-1}$ and in the temperature range from $296 \mathrm{~K}$ to $723 \mathrm{~K}$. At room 
temperature the maximum flow stress at a strain rate of $10^{3} \mathrm{~s}^{-1}$ is about $15 \%$ higher than the stress at $10^{-3} \mathrm{~s}^{-1}$. The dynamic stress-strain behavior of the AZ91 alloy at strain rates between $10^{2} \mathrm{~s}^{-1}$ and $10^{3}$ $\mathrm{s}^{-1}$ was investigated by Han, $\mathrm{Xu}$ and Liu [4]. The flow stress increased at first and then decreased with the strain rate increasing from $10^{2} \mathrm{~s}^{-1}$ to $10^{3} \mathrm{~s}^{-1}$. The alloy exhibited both strain rate hardening and strain rate softening effects within the selected strain rate range. Shu, Zhou and Ma [5] found an increase in the tensile strength of magnesium alloys AM50 with increasing strain rate from $600 \mathrm{~s}^{-1}$ to $1350 \mathrm{~s}^{-1}$. Abbott, Easton and Song [6] investigated the tensile properties of AZ91D in a strain rate range between $0.01 \mathrm{~s}^{-1}$ and $1 \mathrm{~s}^{-1}$ and found no significant effect of strain rate on AZ91D.

From the existing literature, it is observed that the micro-structural characteristics and tensile properties of magnesium alloys at quasi-static loading have been investigated by most of the authors but little is known about its mechanical properties at low to high strain rates between $10^{2} \mathrm{~s}^{-1}$ and $10^{3}$ $\mathrm{s}^{-1}$. In the current study, AZ91D has been investigated under tensile loading using Split Hopkinson Tensile Bar (SHTB) in the range of strain rates between $300 \mathrm{~s}^{-1}$ and $1250 \mathrm{~s}^{-1}$. The strain rate dependence of stress has been evaluated for the alloy.

\section{EXPERIMENTAL SETUP AND DATA ANALYSIS}

The Split Hopkinson Pressure Bar (SHPB) technique is a widely used technique for testing materials at high strain rates. As long as the equipment is calibrated accurately with proper preparations, this technique will offer a relatively high level of accuracy. The tensile Hopkinson bar apparatus consists of one input bar, one output bar, a striker tube, an anvil bar and an absorber bar at the end. Both the input and the output bars are fitted with a pair of strain gauges, each pair mounted $500 \mathrm{~mm}$ away from the specimen end. The strain gauges in a pair are fixed $180^{\circ}$ apart on the bar to record the strain when impacted. The schematic diagram of the tensile Hopkinson bar is shown in Fig. 1

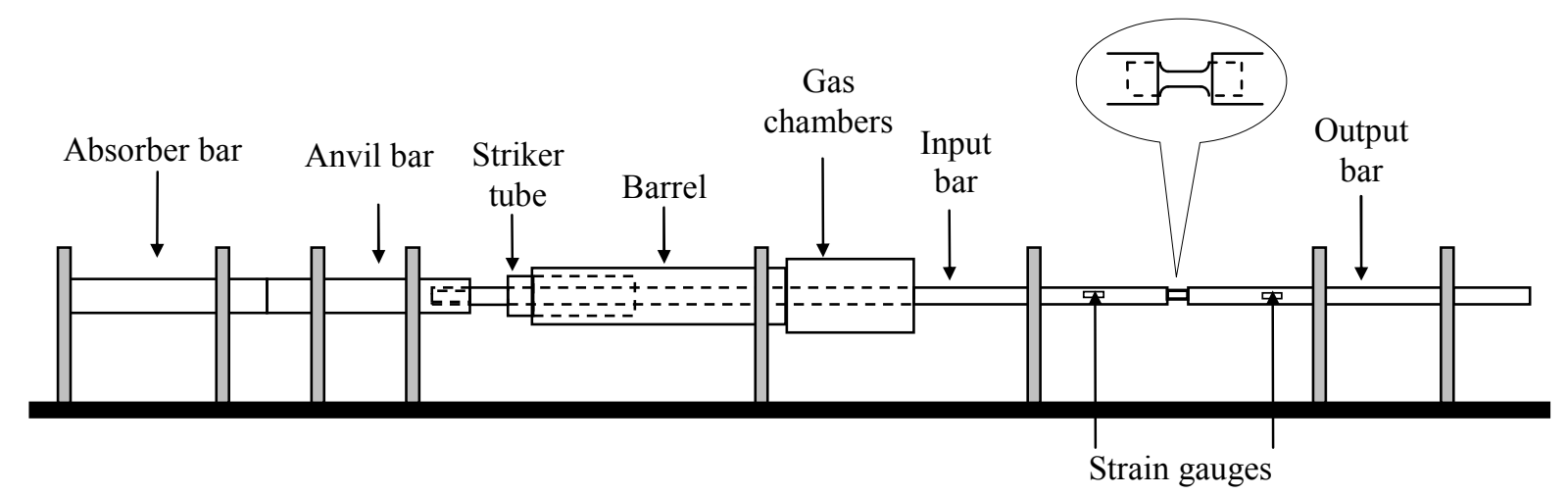

Fig. 1. A schematic diagram of Split Hopkinson Tensile Bar

The dog bone shaped specimen with threaded flanges is screwed between the input bar and the output bar. A gas gun drives the striking tube that is propelled from the barrel. The striking tube hits the anvil bar to initiate a compressive stress wave that propagates with speed $c_{o}$ where $c_{o}=(E / \rho)^{1 / 2}$, being $E$ the elastic modulus and $\rho$ the bar material density, along the anvil bar into the absorbing bar until it reaches the free end of the absorbing bar where it reflects back as a tensile pulse. The tensile pulse will not be able to propagate back into the anvil bar and is absorbed in the absorber bar. A tensile wave also known as the incident wave propagates along the input bar towards the specimen/bar interface where it is measured by the strain gauges on the input bar. At this interface, the incident wave is partially reflected and partially transmitted through the specimen to the output bar as a transmitted wave. The reflected wave is again captured by the strain gauges on the input bar and the transmitted wave is measured by the strain gauges on the output bar. Measuring the amplitude of the incident, reflected and the transmitted pulses, $\varepsilon_{I}, \varepsilon_{R}$ and $\varepsilon_{T}$ respectively, shown in Fig. 2, it is possible to determine the stress-strain relationship of the specimen. 


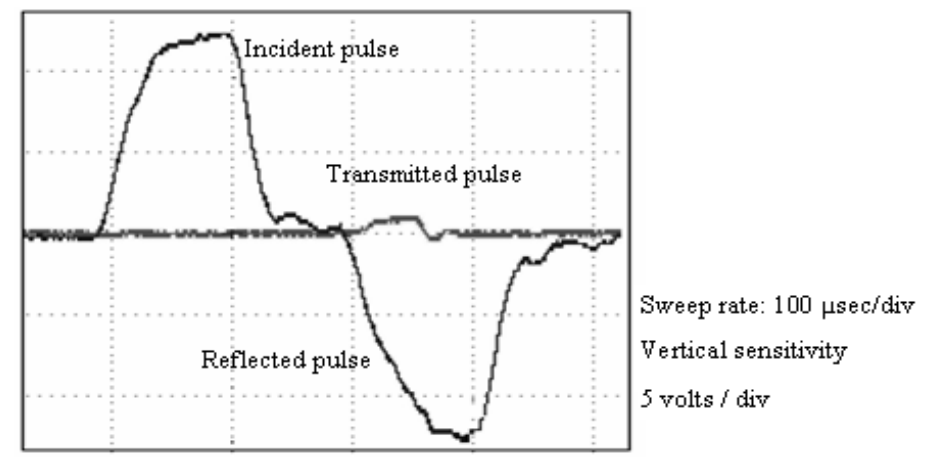

Fig. 2. Oscilloscope traces obtained from tensile Hopkinson bar on AZ91D alloy

When the specimen is deforming uniformly, the strain rate within the specimen is directly proportional to the amplitude of the reflected pulse. Likewise, the stress within the specimen is directly proportional to the amplitude of the transmitted pulse. The strain rate $\dot{\varepsilon}=d \varepsilon / d t$ in the specimen is calculated as

$$
\frac{d \varepsilon_{s}}{d t}=-\frac{2 c_{o}}{l_{s}} \varepsilon_{R} \text {. }
$$

where $l_{s}$ is the length of the specimen before impact. The strain is determined by integrating the strain rate from 0 to $t$, the total duration of the test.

$$
\varepsilon_{s}(t)=-\frac{2 c_{o}}{l_{s}} \int_{0}^{t} \varepsilon_{R}(t) d t
$$

Stress in the specimen can be calculated by using the following equation;

$$
\sigma_{\text {ave }}(t)=E \frac{A_{o}}{A_{s}} \varepsilon_{T}(t)
$$

Where $A_{o}$ and $A_{s}$ are the cross sectional areas of the incident bar and the specimen.

\section{Specimen}

Fig. 3 shows the geometry of the specimens used in the present tests. The specimens were prepared from the same batches of AZ91D alloy to avoid any metallurgical effects.

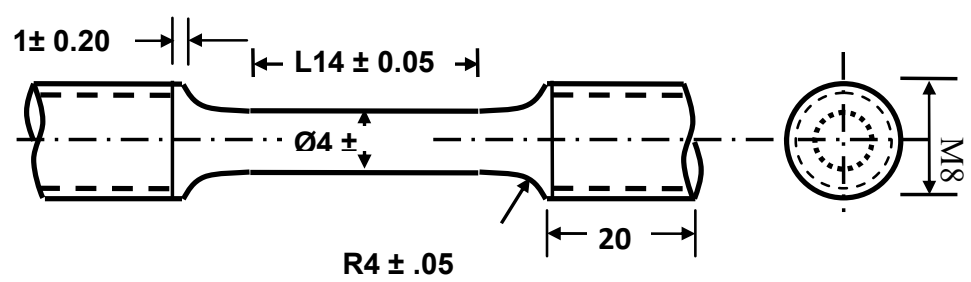

Fig. 3 Dimensions of the tensile specimen used

\section{Test Results and Discussions}

In order to study the tensile behavior of the magnesium alloy AZ91D, a series of impact tests were performed at various strain rates between $300 \mathrm{~s}^{-1}$ and $1250 \mathrm{~s}^{-1}$. The strain rate variation with time is plotted in Fig. 4 for various tested strain rates. The values of strain rates shown in figure 4 are the average values. It is observed that the strain rate is relatively uniform at lower strain rates as compare to high strain rates. 


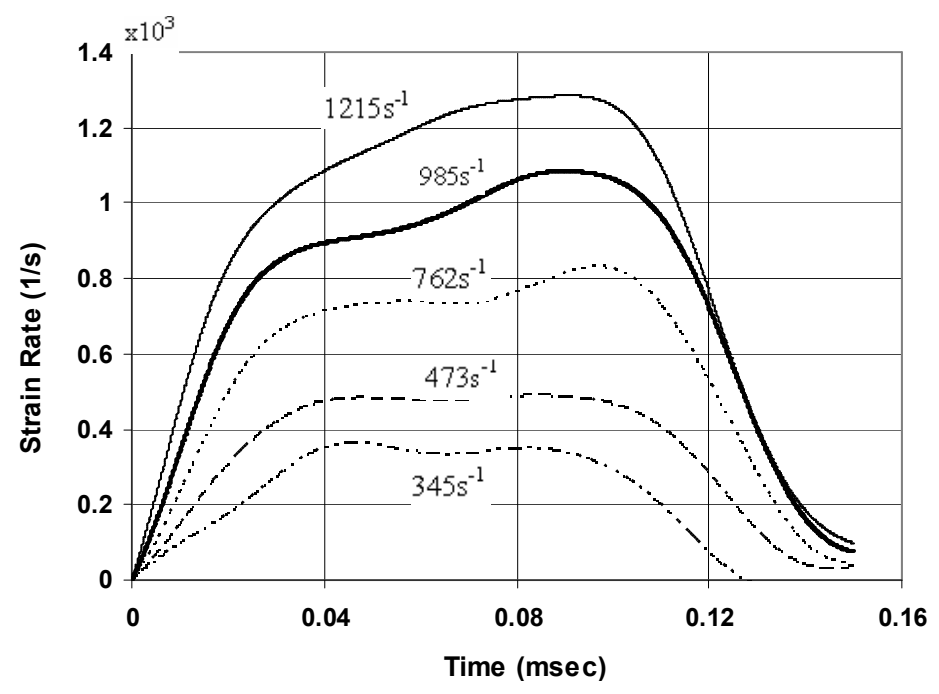

Fig. 4. Strain rate variation with time at different strain rate loadings

\section{Effect of strain rate on dynamic properties of the alloy}

Figure 5 depicts the resulting impact tensile stress-strain relations for the AZ91D alloy at various rates of strain. The alloy exhibit distinct yield points at high strain rates and the stress increases with increasing the strain rate monotonically. At a strain rate of $1215 \mathrm{~s}^{-1}$, approximately $15 \%$ and $45 \%$ high stresses are observed as compare to what is experienced at a strain rate of $345 \mathrm{~s}^{-1}$ at $1.5 \%$ strain and quasi-static test at $2 \%$ strain respectively. A similar trend is seen for other higher strain rates. The elongation to fracture of the specimen increases with increasing strain rate except the specimen tested at $473 \mathrm{~s}^{-1}$. A slight work hardening during plastic deformation is also observed.

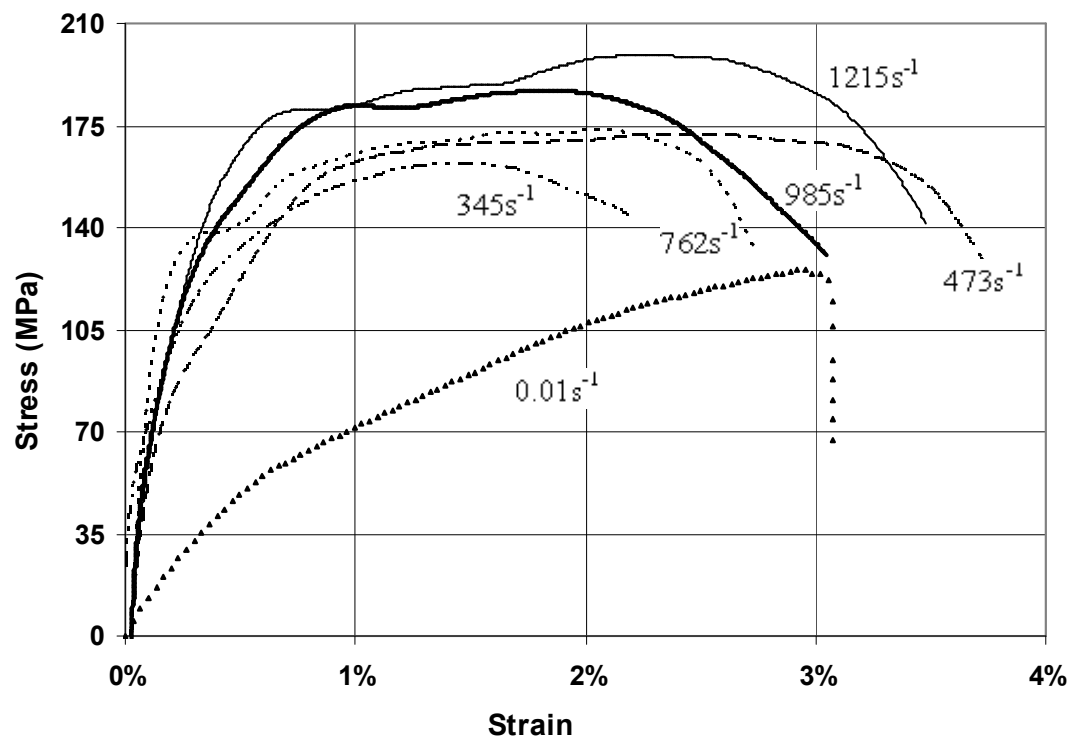

Fig. 5. Stress-strain relation for magnesium alloy AZ91D at different strain rates

\section{Strain-hardening and strain rate sensitivity}

The material deformation becomes increasingly difficult during plastic deformation due to increasing dislocation density. This process is called strain hardening and is depicted by the well known Hollomon equation;

$$
\sigma=K \varepsilon^{n} .
$$

$K$ and $n$ are the strength coefficient and the strain hardening exponent respectively. The hardening exponent can be found by determining the slope of a plot of $\log (\sigma)-v s-\log (\varepsilon)$. Figure 6 , shows such 
a plot for the current study at various strain rate levels. The value of $n$ calculated from this plot for three different strain rates 345,762 and $1215 \mathrm{~s}^{-1}$ are $0.23,0.196$ and 0.185 indicating a slight decrease in the hardening exponent with increasing strain rate. In order to examine the effect of the strain rate on the tensile stress, the respective values of stress at different percentage strains are plotted in Fig. 7 as a function of log of average strain rate. In order to evaluate the rate sensitivity of the stress the strain rate sensitivity $m$, is introduced in the following equation;

$$
m=\left(\frac{\partial \sigma}{\partial \log \dot{\varepsilon}}\right)_{\varepsilon, \mathrm{T}} .
$$

Where $\mathrm{T}$ is the test temperature, which is room temperature in the present study

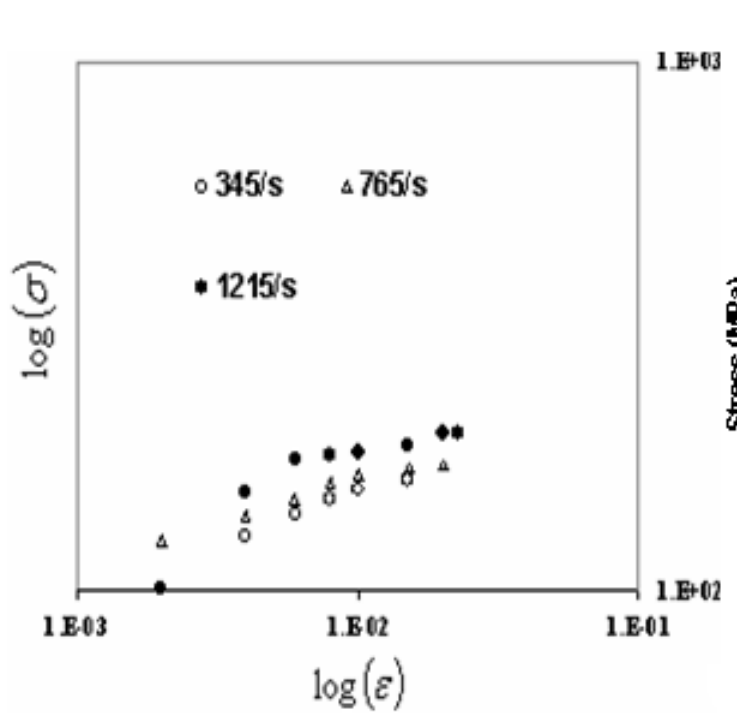

Fig. 6. $\log (\sigma)$-vs-log $(\varepsilon)$

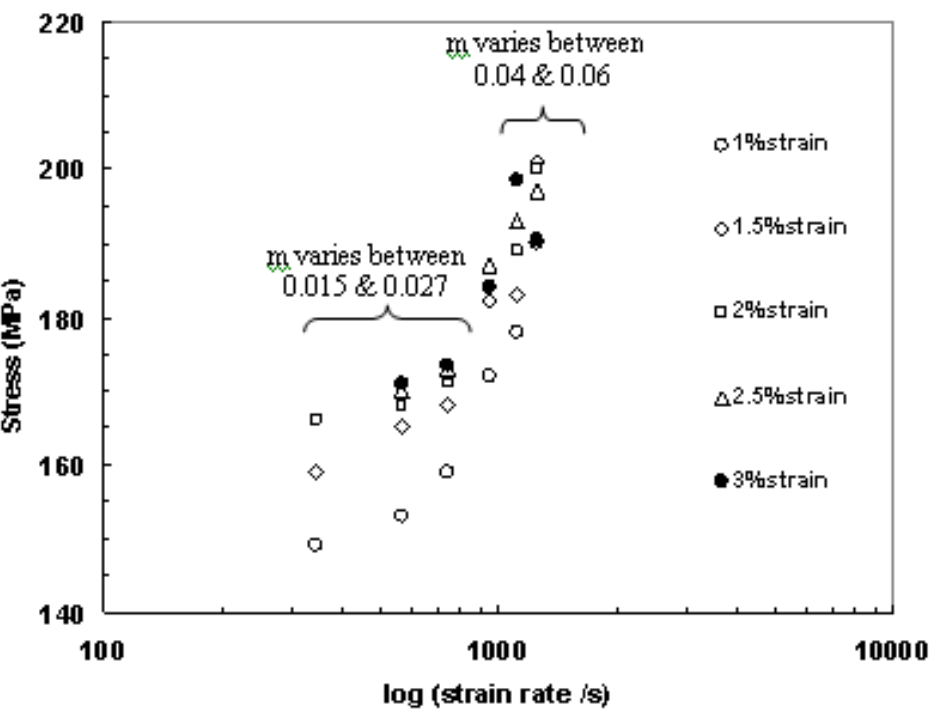

Fig. 7. Stress as a function of log strain rate.

The stress $\sigma$ corresponds to different strain rates at some constant strain $\varepsilon=\varepsilon_{\circ}$. The value of $\mathrm{m}$ is plotted for different values of specimen strain. It is clear that the alloy AZ91D is a rate sensitive material. It is noted that the strain rate sensitivity of the AZ91D alloy varies between 0.015 and 0.027 for strain rate lower than $1000 \mathrm{~s}^{-1}$ and it is between 0.04 and 0.06 when the deformation rate is higher than $1000 \mathrm{~s}^{-1}$ indicating high rate sensitivity of the alloy at higher strain rates. The values of strain rate sensitivity calculated for the AZ91D alloy for strain rates up to $1000 \mathrm{~s}^{-1}$ are plotted as a function of the specimen strain in Fig. 8. As the strain increases, the strain rate sensitivity decreases monotonically. However, the decrease in strain rate sensitivity is minute for larger strains $(>2 \%)$ as compare to what is observed at lower strains. Similar behavior is observed for strain rate higher than $1000 \mathrm{~s}^{-1}$.

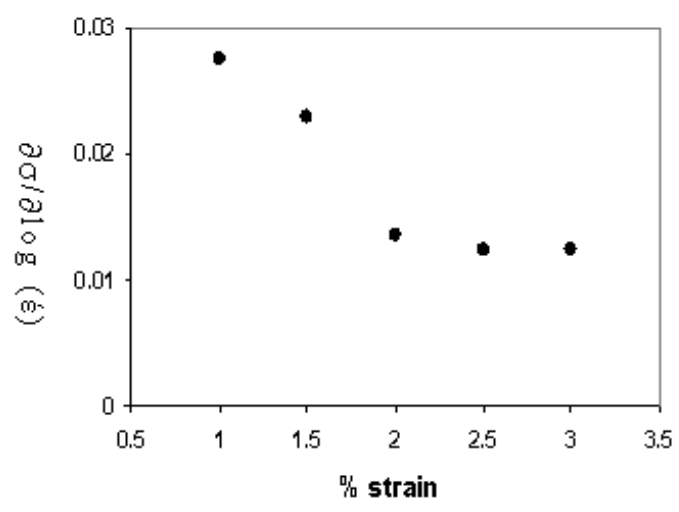

Fig. 8. Strain rate sensitivity $m$ vs. specimen strain 


\section{CONCLUSION}

A die cast magnesium alloy AZ91D has been investigated for its tensile properties at high strain rates. It is observed that the strain rate variation with time is relatively uniform when the specimen is deforming at lower strain rates as compare to high strain rates. A monotonic increase in the stress is observed with increasing strain rate from $345 \mathrm{~s}^{-1}$ to $1215 \mathrm{~s}^{-1}$. At a strain rate of $1215 \mathrm{~s}^{-1}$, approximately $15 \%$ and $45 \%$ high stresses are observed as compare to what is experienced at a strain rate of $345 \mathrm{~s}^{-1}$ at $1.5 \%$ strain and quasi-static test at $2 \%$ strain respectively. The hardening exponent decreases with the increasing the strain rate. It is noted that the strain rate sensitivity for AZ91D alloy is lower for strain rate up to $10^{3} \mathrm{~s}^{-1}$ and it increases sharply at strain rates higher than $10^{3} \mathrm{~s}^{-1}$. A decrease in the strain rate sensitivity is observed with increasing \%strain in the specimen.

\section{Future Recommendations}

More tests with an extended range of strain rates up to 2000 and higher followed by numerical simulation using the Johnson-Cook materials mode, needed for better understanding of the dynamic behaviour of the alloys, are in progress.

\section{Reference}

1. Kainer, K., U. Magnesium-Alloys and Technologies. WILEY-VCH, Weinheim, 2003.

2. Aune, T. Kr., Darryl Albright, Hakon Westengen, Jojnsen Tor E., Andersson B., "Behavior of Die Cast Magnesium Alloys Subject to Rapid Deformation," 2000-01-1116, SAE International (2000)

3. Ishikawa, K., Watanabe, H. and Mukai, T., "High strain rate deformation behavior of an AZ91 magnesium alloy at elevated temperatures," Materials Letters (59), 1511-15 (2005)

4. Han, E. H., Xu, Y. B. and Liu, L., "Dynamic stress-strain behavior of AZ91 alloy at high strain rate," Materials Science Forum 546-549(1), 89-92 (2007)

5. SHU, D. W., Zhou, W. and MA, G. W., "Tensile Mechanical Properties of AM50 Alloy by Hopkinson Bar, Key Engineering Materials," (340-341), 247-254 (2007)

6. Song, W. Q., Beggs, P. and Easton, M., "Compressive strain-rate sensitivity of magnesiumaluminium die casting alloys," Materials and Design (30), 642-648 (2009)

7. Nicholas, T., "Tensile Testing of Materials at High Rates of Strain," Experimental Mechanics, (21), 177-185 (1980)

8. Staab, G.H. and Gilat, A., “A Direct-tension Split Hopkinson Bar for High Strain-rat Testing," Experimental Mechanics, (31), 232-235 (1991) 